\title{
FUNGAL DEGRADATION OF LIGNIN-BASED RIGID POLYURETHANE FOAMS
}

\author{
Sepúlveda, M. ${ }^{1}$, Fernandes, I.P. ${ }^{1}$, Amaral, J.S. ${ }^{2}$, Barreiro, M.F. ${ }^{1}$ \\ 1. LSRE/IPB, Campus de S. Apolónia Ap 1134, 5301-857 Bragança, Portugal (barreiro@ipb.pt) \\ 2. REQUIMTE/FFUP, Rua Aníbal Cunha, 164, 4099-030 Porto, Portugal and IPB, Campus de Santa Apolónia \\ Ap 1134, 5301-857 Bragança, Portugal (jamaral@ipb.pt)
}

\begin{abstract}
Introduction
The environmental impacts arising from the use of polymeric materials is a serious problem since they can present long periods of degradation and hence being accumulated in the environment. Polyurethanes (PU) are considered as one of the most versatile polymeric materials offering a wide range of products with applications in diverse sectors. Rigid polyurethane (RPU) foams, due to its excellent insulation and mechanical properties, are widely used in construction, automotive, freeze industry and nautical applications. In this context, the use of green polyols with value-added properties, such as biodegradability, will contribute to reduce environmental impacts.

In this work the ability of Aspergillus niger to degrade lignin-based RPU foams in comparison with a control (RPU foam based on a commercial polyol), was tested according to various procedures.
\end{abstract}

\section{Materials and methods}

Test samples: Two RPU foams designated as "Indulin AT" and "Alcell", following the technical lignins used to produce the lignin-based polyols by oxypropylation [1], were evaluated. A RPU foam based on a commercial polyether polyol (Lupranol ® 3323) was used as control and designated as "Commercial". Samples were cut into blocks (6.0x6.0 $\mathrm{mm}$ and $2.0 \mathrm{~mm}$ thickness) and weighted before being tested.

Biodegradation assays: Different tests were performed to evaluate samples degradation, namely (i): degradation on agar plates, by sample inoculation with Aspergillus niger (ATCC 16404) on a nutrient agar plate and incubation during 28 days $/ 30^{\circ} \mathrm{C}$; (ii): Respirometry in liquid-media, based on ISO 14852:1999 with modifications and (iii): Respirometry/soil assays, based on ISO 17556:2003, with adaptations. In (ii) and (iii) a series of batch cultures were incubated at $30^{\circ} \mathrm{C}$ and the $\mathrm{CO}_{2}$ produced was periodically monitored during 90 days.

\section{Results and discussion}

In this work, degradation tests were performed using A. niger since the ability of this fungi to degrade flexible polyurethane foams has already been reported [2]. First, to evaluate if the foams could inhibit the fungi growth, agar plate tests were performed. Visual observations allowed verifying the fungi growth on foams surface as well as the appearance of dark spots (fungi spores) both in the surface and interior. After 28 days, the samples were observed by optical microscopy (OM) and it was possible to observe the presence of hifae and spores in the RPU foam cells. Figure 1 presents an example of the images obtained for the Alcell sample. Considering that, in aerobic conditions, $\mathrm{CO}_{2}$ is one of the major metabolites arising from microorganism's carbon oxidation, its determination can be correlated to polymer biodegradation [3]. Thus, respirometry tests (in liquid media and soil) were performed to evaluate foams degradation based on the $\mathrm{CO}_{2}$ produced. First, liquid media respirometry tests were performed during 90 days using nutritive both $(13 \mathrm{~g} / \mathrm{L})$ inoculated with $1 \mathrm{~mL}$ of a suspension containing approximately $1 \times 10^{6}$ spores of $A$. niger. However, since the used media was very rich in nutrients, a strong growth of $A$. niger occurred in all flasks and only slight $\mathrm{CO}_{2}$ differences were observed for the 3 foams under evaluation. Consequently, the same test was repeated with diluted nutritive broth (1:1000) in order to provide less nutrients and induce the foam degradation by the microorganisms. As expected, a weaker development of the fungi and a consequent lower $\mathrm{CO}_{2}$ production were observed for all samples. As can be observed in Figure 2, Indulin AT was the foam who presented a higher $\mathrm{CO}_{2}$ production, followed by Alcell and finally by the commercial foam. Identical results were observed in the previous test, however the differences among samples were now more clear. In the OM visualization of the foams, the presence of fungi hifae was observed and 
degradation was evidenced by changes in the cell wall structures, with surface irregularities, small holes and cracks (Figure 3). The soil assays were performed with and without A. niger addition. In both the obtained results were identical to the ones obtained in the liquid media respirometry assays, confirming that the lignin-based foam Indulin AT seems to be less resistant to fungi degradation.
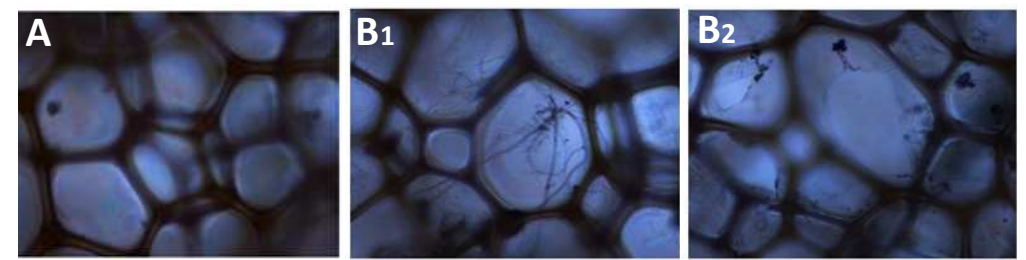

Figure 1. Optical microscopy images (100x) of the agar plate test for Alcell foam (A): non-inoculated and (B): after inoculation with A. niger and 28 days of incubation at $30^{\circ} \mathrm{C}$.

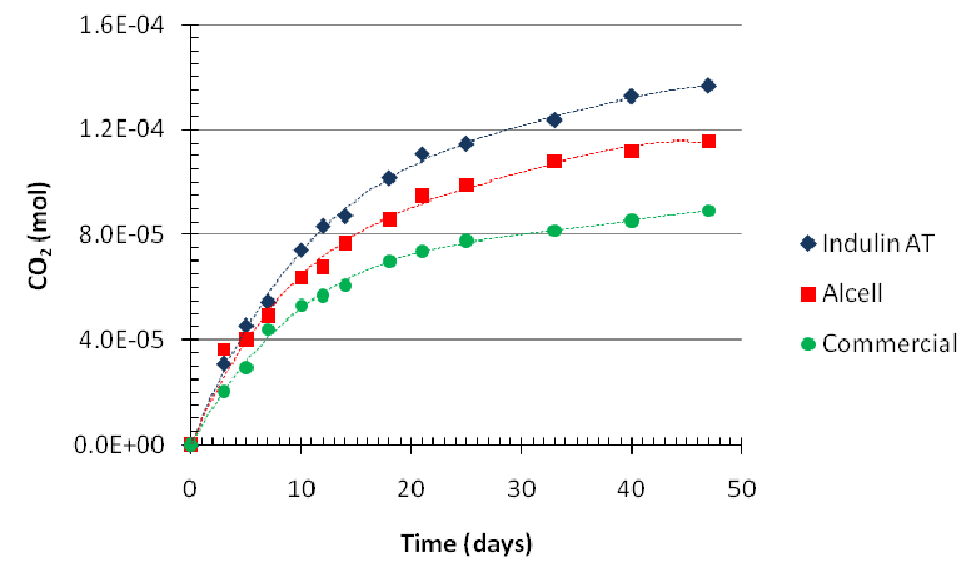

Figure 2. Respirometry results in liquid-media assays using diluted nutritive broth (1:1000).

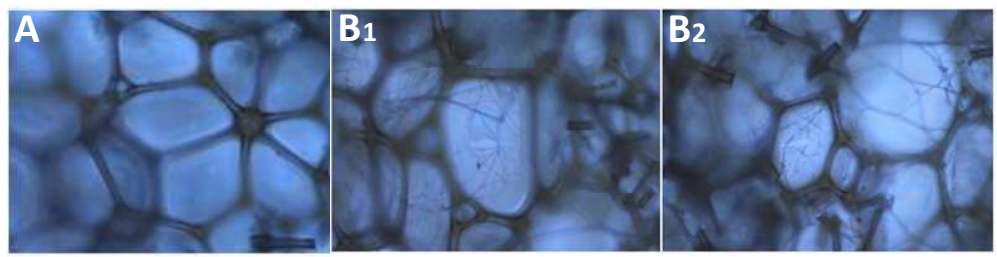

Figure 3. Optical microscopy images (100x) of the respirometry test in diluted nutrient broth $(1: 1000)$ for Indulin AT foam (A): non-inoculated and (B): after inoculation with A. niger and 60 days at $30^{\circ} \mathrm{C}$.

\section{Conclusions}

Currently, the information concerning microbial degradation of PU in the environment is still limited. In this study, complete polymer biodegradation did not occurred, but PU foam degradation by A. niger fungi was observed. Both lignin-based PU foams presented higher degradation when compared to the commercial PU foam.

\section{Acknowledgements}

The used lignin-based RPU foams were synthesized within the context of Carolina Cateto's PhD thesis (Lignin-Based Polyurethanes:Synthesis, Characterization and Applications). Financial support from FCT (project PTDC/CTM/71491/2006_FCOM-01-0124-FEDER-007156) is acknowledged.

\section{References}

[1] C.A. Cateto, M.F. Barreiro, A.E. Rodrigues, M.N. Belgacem Ind. Chem. Res. 2009, 48, 2583.

[2] M.H. Alma; M. A. Basturk J. Mater. Sci. Lett. 2003, 23, 1225.

[3] A.A. Shah; F. Hasan; A. Hameed; S. Ahmed Biotechnol. Adv. 2008, 26, 246. 